\title{
ANQUILOSE TEMPOROMANDIBULAR BILATERAL: ASPECTOS FONOAUDIOLÓGICOS E PROCEDIMENTOS CLÍNICOS
}

\author{
Bilateral ankylosis of the temporomandibular joint: \\ oral miofunctional aspects and clinical procedure
}

\author{
Silvana Regina Marzotto ${ }^{(1)}$, Esther Mandelbaum Gonçalves Bianchini ${ }^{(2)}$
}

\begin{abstract}
RESUMO
Objetivo: apresentar uma proposta terapêutica miofuncional orofacial, os procedimentos utilizados e resultados em caso de anquilose temporomandibular bilateral, buscando ressaltar a importância do trabalho interdisciplinar e a contribuição da fonoaudiologia para a reabilitação do sistema estomatognático nesses casos. Métodos: relato de caso de paciente adulto, gênero masculino, encaminhado para avaliação e procedimentos fonoaudiológicos após três meses da realização de cirurgia articular, tendo como queixa principal grande restrição na abertura da boca. Na avaliação fonoaudiológica foi constatado: abertura da boca máxima em $25 \mathrm{~mm}$, ausência de movimentos de lateralidade e de protrusiva, mordida aberta anterior em $8 \mathrm{~mm}$, dor constante em região temporal, dificuldade para realizar mastigação, deglutição, articulação da fala. Constou dos objetivos terapêuticos: estimular e possibilitar a execução de movimentos mandibulares minimizando progressão de tecido cicatricial restritivo e reabilitação miofuncional. Procedimentos quanto ao aumento da amplitude através de relaxamentos, alongamentos, massagens, movimentos isotônicos, isométricos, isocinéticos e manobras específicas foram essenciais para estabilizar a abertura da boca, liberar os movimentos mandibulares e coordená-los, meIhorando a funcionalidade do sistema estomatognático. Resultados: após 24 sessões: ganho de 16 $\mathrm{mm}$ na abertura passando para $41 \mathrm{~mm}$; redução da mordida aberta anterior para $4 \mathrm{~mm}$, melhora da higiene oral, da articulação da fala, das condições alimentares com ganho de força mastigatória e redução dos mecanismos compensatórios; eliminação da dor em região temporal, além de melhora na estética facial. Conclusão: a terapêutica fonoaudiológica miofuncional orofacial mostrou-se eficiente em adulto com anquilose temporomandibular bilateral pós-cirúrgico, com estabilidade dos resultados mantida segundo controle anual.
\end{abstract}

DESCRITORES: Anquilose; Articulação Temporomandibular; Sistema Estomatognático; Traumatismos Faciais; Reabilitação

\section{INTRODUÇÃO}

A Articulação Temporomandibular (ATM), estrutura do sistema estomatognático, é uma das mais complexas e dinâmicas dentre as articulações do corpo

(1) Fonoaudióloga Clínica, CEFAC - Saúde e Educação - Setor de Distúrbios da ATM, Cirurgia Ortognática e Traumas de Face.

(2) Fonoaudióloga, Professora do CEFAC - Saúde e Educação; Doutora em Ciências - Fisiopatologia Experimental pela Faculdade de Medicina da Universidade de São Paulo. humano. AATM tem a capacidade de propiciar movimentos diferentes bilateralmente, porém com dinâmica de unidade. Por ser uma articulação bilateral, o acometimento de um dos lados acaba modificando o funcionamento do outro ${ }^{1}$.

Diante de alterações na ATM, irão ocorrer prejuízos ao sistema estomatognático no que diz respeito ao desenvolvimento das funções, em especial, a mastigação, uma vez que a mesma é totalmente dependente do movimento mandibular ${ }^{2}$. Dentre as diversas anomalias que afetam a articulação temporomandibular, a anquilose, afecção 
de prognóstico muito reservado, pode representar um comprometimento social e psicológico muito intenso ao paciente, devido às alterações funcionais e estéticas ${ }^{3}$.

A etimologia do termo anquilose refere-se à aderência em articulação. Devido à aderência da articulação com conseqüente rigidez entre as suas superfícies, os movimentos mandibulares encontram-se impossibilitados ou limitados. Sua gravidade está diretamente relacionada com o tipo de extensão da lesão, a época do acometimento devido à possibilidade de interferência nas etapas de crescimento e o tempo transcorrido sem tratamento. A etiologia é principalmente relacionada a trauma direto na ATM ou indireto, sendo encontradas também referências a quadros infecciosos e inflamatórios maxilares ou articulares graves: artrites reumáticas e causas congênitas ${ }^{4-6}$.

Uma proposta de classificação para anquilose da ATM foi apresentada, dividindo-a em dois tipos: anquilose verdadeira e anquilose falsa ${ }^{7}$. Correntemente, a anquilose intra-articular (intracapsular) é chamada de anquilose verdadeira e a extra-articular (extra-capsular), de anquilose falsa ${ }^{5,8-11}$. A anquilose falsa é devida à rigidez das partes que rodeiam a articulação. A verdadeira é devida à união, ou seja, fusão dos ossos de uma articulação móvel. Anquilose intra-articular e extra-capsular podem ocorrer juntas ${ }^{5}$. A forma intraarticular é mais freqüente e tem tendência a transformar-se em extra-articular se for deixada sem tratamento por um longo tempo ${ }^{12}$.

A terminologia também pode referir-se ao tipo de tecido que promove a limitação do movimento mandibular, ou seja, a anquilose pode ser fibrosa ou óssea ${ }^{10,13,14}$. Na anquilose óssea os fatores limitantes da mobilidade mandibular, quer sejam intra ou extra-articulares, são de tecido duro, e a ATM pode ser substituída por massa óssea ${ }^{13,15}$. A anquilose óssea representa a união da cabeça da mandíbula com o osso temporal, acarretando total impedimento do movimento mandibular ${ }^{16}$. $\mathrm{Na}$ anquilose fibrosa, geralmente extra-articular, a limitação dos movimentos da mandíbula é por formação de bridas de origem cicatricial ou muscular ${ }^{17,18}$. A deformidade facial será assimétrica quando a anquilose for unilateral e simétrica quando for bilateral ${ }^{18,19}$. O acometimento bilateral é mais raro e de pior prognóstico. Nesses casos ocorre também grande prevalência de mordida aberta anterior, devido à perda bilateral de altura posterior do ramo ascendente da mandíbula, após a remoção cirúrgica do segmento anquilosado, modificando a situação oclusal e abrindo a mordida anteriormente ${ }^{20}$. A anquilose óssea é de maior importância devido aos problemas funcionais que o paciente apresenta, além da dificuldade de tratamento.

Os resultados apresentados em trabalhos sobre a avaliação das funções estomatognáticas em sujeitos com anquilose temporomandibular confirmam que a função mais comprometida nestes pacientes é a mastigação e, em decorrência disso, há uma grande dificuldade para a alimentação 18,19. Embora o maior problema resida nas restrições funcionais referentes à mastigação, deglutição e fala; a estética facial fica bastante prejudicada devido à micrognatia e/ou assimetria facial intensa $2,5,19,20$.

Em síntese, a anquilose pode ser definida como a limitação crônica de movimento de uma articulação. Pode ser parcial ou total; fibrosa ou óssea; intraarticular (verdadeira) ou extra-articular (falsa) e unilateral ou bilateral, podendo ocorrer combinações entre estes dois tipos ${ }^{14}$.

O diagnóstico é feito com base nos sinais e sintomas da anquilose e confirmados por exames de imagem. O detalhado exame clínico e anamnese são de grande importância para levantarmos hipótese sobre o tipo de comprometimento articular. Os exames radiográficos, tomografia e/ou ressonância nuclear magnética definem o tipo e a extensão da lesão mostrando os limites anatômicos preservados, presença de alterações e de tecido ósseo radiopaco ou outras alterações que dificultem ou impeçam os movimentos livres da mandíbula ${ }^{3,14}$.

O tratamento da anquilose da ATM inicialmente é cirúrgico e se baseia em excisão adequada e ressecção da anquilose seguida de fisioterapia ${ }^{3,19}$ ou terapia fonoaudiológica miofuncional oral ${ }^{20-22}$. São muitas as técnicas desenvolvidas e descritas para a correção cirúrgica da anquilose temporomandibular. Independente da técnica adotada, o objetivo único é a liberação da mandíbula do paciente proporcionando a funcionalidade desta complexa estrutura. São verificadas descrições de cirurgias com grande incisão pré-auricular, ressecção da área anquilosada, rebatendo-se parte do feixe médio do músculo temporal para preenchimento da região ${ }^{6,8-10,16-20}$

Visando a recuperação da função, o acompanhamento pós-operatório por meio de drenagem do edema, a orientação de exercícios e o direcionamento funcional são tão importantes quanto o próprio procedimento cirúrgico, devido à possibilidade de recidiva do problema ${ }^{3,13,19-29}$.

É comum a ocorrência de assimetria facial óssea e muscular, alterações dentárias que levam a limitações funcionais, mesmo depois do tratamento cirúrgico da região acometida. A musculatura atrofiada pela falta de uso em virtude de restrição óssea, precisa ser intensamente estimulada após 
a cirurgia. É necessário iniciar, em curto prazo, a mobilização mandibular para direcionar a formação de tecido cicatricial, sem permitir que esta restrinja o movimento ${ }^{19,20,30-32}$.

Alguns autores ${ }^{30}$ defendem que os exercícios devam ser iniciados 24 horas após a cirurgia mesmo que a dor presente seja um fator de desestímulo. Os treinos mioterápicos devem ser concomitantes à reabilitação funcional, implícito em acompanhamento posterior para manutenção dos resultados obtidos por ao menos um ano ${ }^{31-33}$.

Na prática clínica fonoaudiológica, constatase, que os casos de anquilose temporomandibular, mesmo já operados passam novamente a apresentar restrição de amplitude de abertura mandibular devido à nova formação de tecido cicatricial e grande dificuldade funcional. A mastigação, uma das funções mais importantes do sistema estomatognático, é a mais prejudicada ${ }^{20,21,31,32}$. Os pacientes realizam a função da mastigação com muita dificuldade, usando mecanismo adaptativo de estruturas associadas. A deglutição mostrase compensada com elevação brusca da laringe e ou movimento associado de cabeça com grande função de dorso da língua. A articulação dos sons da fala apresenta-se com reduzida amplitude do movimento mandibular, ressonância posterior e pobreza de articulação comprometendo a inteli-gibilidade ${ }^{21,31-33}$.

A proposta fonoaudiológica, por meio de reabilitação miofuncional orofacial, visa principalmente o direcionamento da mobilidade da mandíbula, alongamento do tecido cicatricial, imediata, estimulação da musculatura facial e reabilitação funcional. Os exercícios buscam o estímulo à musculatura facial e os treinos o restabelecimento das funções estomatognáticas, em especial a mastigação, deglutição e fala ${ }^{31-33}$.

O tratamento da anquilose temporomandibular deve, portanto, ser multidisciplinar. A interdependência entre equipes: cirurgiões, ortodontistas, fonoaudiólogos e fisioterapeutas, são fundamentais para viabilizar e restabelecer a funcionalidade do sistema estomatognático, dentro das limitações de prognóstico que muitos casos determinam.

Assim, o objetivo deste trabalho foi apresentar uma proposta terapêutica miofuncional orofacial e os procedimentos utilizados em caso de anquilose temporomandibular bilateral, buscando ressaltar a importância do trabalho interdisciplinar e a contribuição da fonoaudiologia para a reabilitação do sistema estomatognático nesses casos.

\section{MÉTODOS}

Trata-se de estudo retrospectivo, por meio de relato de caso, com dados do prontuário, da avaliação fonoaudiológica e descrição das técnicas de terapia fonoaudiológica miofuncional utilizadas durante atendimento realizado na Clínica Escola CEFAC Assistencial (ONG CEFAC).

\section{Relato de Caso}

Paciente adulto, sexo masculino, 28 anos, com diagnóstico de anquilose temporomandibular bilateral definido pela equipe odontológica. A etiologia foi dada como desconhecida. Apresenta histórico de dificuldade para alimentar-se desde criança e impossibilidade de tratamento dentário. Segundo dados de prontuário do paciente, foi realizado procedimento cirúrgico de artroplastia e discoplexia, para obtenção de uma articulação funcional com a criação de uma pseudo-artrose pela ressecção do bloco ósseo de anquilose. Apresentava abertura mandibular máxima prévia à cirurgia de $17 \mathrm{~mm}$. Após três meses da realização da cirurgia, o paciente foi encaminhado para o Setor de Distúrbios da ATM, Cirurgia Ortognática e Traumas de Face da ONG CEFAC, por uma fonoaudióloga, para avaliação e procedimento fonoaudiológico miofuncional orofacial.

Os procedimentos fonoaudiológicos seguiram protocolos específicos de anamnese e exame fonoaudiológico para traumas de face ${ }^{31,32}$.

Quanto aos dados de anamnese obtivemos: queixa de dor na região de músculo temporal esquerdo, presença de ruído articular sugestivo de crepitação durante abertura mandibular, grave limitação dos movimentos mandibulares, ausência de sintomas auditivos e labirínticos, falta de força para mastigar e conseqüentemente dieta alimentar mais pastosa. Não foram relatados comprometimentos em outras articulações. Negou hábitos orais nocivos.

A radiografia panorâmica pré-cirúrgica apresentada mostrou massa radiopaca de densidade variável e união do osso temporal com o osso mandibular bilateralmente.

$\mathrm{Na}$ avaliação fonoaudiológica constatou-se grave desproporção dos terços da face, sendo maior o inferior, e significante assimetria facial com desvio do mento para o lado esquerdo. Edema bilateral próximo à região operada ainda presente meso após três meses da cirurgia. No aspecto intra-oral observouse maloclusão acentuada com mordida cruzada bilateral, palato atrésico e mordida aberta anterior de $8 \mathrm{~mm}$. Desvio da linha média para o lado esquerdo, ausência de elementos dentários (primeiro e segundo molares inferiores esquerdos), visível interferência oclusal, mobilidade de elementos dentários (incisívos inferiores), desproporção entre maxila e mandíbula, maxila atrésica e assoalho profundo. 
Quanto às características funcionais o paciente apresentou: respiração predominantemente nasal, mastigação apenas de alimentos semi-sólidos estando desorganizada, com movimentos lentos, reduzidos e unicamente verticalizados com ausência de lateralidade mandibular. Observou-se uso excessivo de estruturas peri-orais associadas e amassamento do alimento com utilização do dorso da língua. Articulação da fala com distorção em fones fricativos sibilantes, intensidade baixa e prejuízo na inteligibilidade. Compensações musculares para a realização da fala, mastigação e deglutição, principalmente por apoio da musculatura supra-hiódea, infra-hióidea e cervical. Higienização precária em função da restrição em abertura da boca. Abertura mandibular máxima em 25 mm, com grande esforço e utilização excessiva da musculatura supra e infrahióidea, assim como apoio do músculo platisma.

As características musculares observadas foram: músculo mentual em hiperfunção para a obtenção do selamento labial, secundário ao aumento do terço inferior da face; músculos masseteres delgados, com massa muscular muito reduzida e pouca força; dor à palpação nos músculos temporais direito e esquerdo; musculatura supra-hióidea rígida; redução da força da língua.

Apesar do caso ter sido encaminhado para terapêutica fonoaudiológica tardiamente em relação ao preconizado na literatura ${ }^{31-33}$, ou seja, três meses após a cirurgia; a proposta fonoaudiológica foi iniciada imediatamente após a avaliação. Foi explicado ao paciente a necessidade de treinos assíduos, assim como o prognóstico possível para esse prazo de início terapêutico.

A abordagem terapêutica miofuncional orofacial baseou-se na aplicação do protocolo específico para anquilose temporomandibular ${ }^{32}$, adaptado para casos bilaterais, cuja proposta será aqui detalhada.

Constaram no planejamento os seguintes objetivos:

- Estimular e possibilitar a execução de movimentos mandibulares, impedindo a formação de tecido cicatricial restritivo.

- Estimular a musculatura mastigatória, atrofiada pela falta de uso, promovendo oxigenação, mobilidade coordenação e aumento de massa.

- Estimular movimentos de abertura mandibular, lateralidade e protrusiva.

- Aumentar a abertura da boca por meio de manobras específicas de alongamentos e alavancas.

- Reabilitação funcional quanto á fala, mastigação e deglutição.

Os registros da amplitude dos movimentos mandibulares foram sempre realizados em todas as sessões, ao início e término de cada sessão, buscando-se constatar a eficiência dos procedimentos realizados em cada terapia, assim como a progressão dos resultados.

Constaram das sessões, os seguintes procedimentos:

I - Conscientização e propriocepção dos aspectos estruturais e funcionais.

II - Preparo da região escapular, cervical, facial e musculatura específica: temporais, masseteres, esternocleidomastóideos por meio de:

- alongamento e estimulação da musculatura do pescoço através de movimentos de lateralização e inclinação da cabeça;

- termoterapia: aquecimento com aplicação de compressas mornas úmidas próximas à área operada e com edemas por tratar-se de caso tardio;

- drenagem manual dos edemas bilateralmente, visando aceleração da recuperação do processo inflamatório e aumento do nível de oxigenação dos tecidos por meio de movimentos de compressão leves e descendentes:

- alongamento por meio de massagens externas em região dos músculos temporais e masseteres, contrária à direção de contração das fibras, bilateralmente. Alongamento dos músculos masseteres com massagem bidigital (interna e externa concomitante) em deslizamento diagonal e finalmente, alongamento por vibração bidigital, tracionando o músculo em direção anterior e inferior; bilateralmente, realizados unilateralmente a cada vez;

- massagem rotatória e deslizamento bidigital em musculatura supra-hióidea, com apoio em mandíbula, unilateral a cada vez, induzido a lateralidade mandibular.

III - Movimentos preparatórios.

Mobilidade dirigida da musculatura perioral por meio de movimentos isotônicos, constando de:

- movimentos de protrusão e retração labial;

- movimentos de lateralidade labial para os dois lados;

- contração de bucinadores contra resistência do dedo colocado internamente;

- movimentos de língua seqüenciais de propulsão e retração; máxima elevação e abaixamento de ponta em protrusão máxima, visando abertura espontânea da boca;

- rotação e lateralização de língua no arco inferior com esforço em amplitude máxima, visando o movimento associado da mandíbula.

Foram propostas duas séries seguidas de 20 movimentos para os exercícios de lábios e bochechas, e de 10 movimentos para os de língua, devido ao esforço necessário para esses últimos.

IV - Movimentos mandibulares.

- Exercícios isotônicos: de abertura e fechamento; protrusivo e retrusivo através da indução do contato incisal inferior com lábio superior e de lateralidade 
com o auxílio da rotação da língua, em seqüências de 10 movimentos com máximo de esforço possível.

- Aumento da abertura máxima, mesmo com desvio, mantendo a mandíbula no limite da maior amplitude obtida por alguns segundos. Por tratarse de caso tardio, pode ser mantido apoio e pressão do dedo polegar nos incisivos superiores e dedo indicador nos incisivos inferiores, com abaixamento da parte posterior da língua e inspiração pela boca associada a esforço da abertura na tentativa de estimular o bocejo, em torno de 5 segundos. Cabe salientar que em casos de procedimento cirúrgico bilateral recente esse tipo de alavanca em região anterior deve ser evitado devido à direção de esforço e redução de altura posterior que essa manobra direciona.

- Abertura forçada com o recurso das espátulas apoiadas entre os dentes posteriores, bilateralmente, alternando-se os lados ou simultaneamente por ao menos 2 a 3 minutos.

- Manobras de distração com o apoio das espátulas colocadas bilateralmente nos dentes posteriores; movimento de alavanca com apoio das espátulas, elevandose a ponta livre dos palitos em direção superior.

A utilização da manobra com espátulas intraoral, em situação pós-cirúrgica da cabeça da mandíbula ou fratura da mesma, só deve ser realizada com apoio das espátulas em região dos últimos dentes molares presentes do paciente. $O$ apoio e pressão realizados no sentido superior, ou seja, de baixo para cima, busca alongamento em região posterior e ganho de altura posterior da mandíbula. As espátulas nunca devem ser colocadas na região anterior, uma vez que a utilização nessa posição leva à redução de altura funcional posterior devido à direção da alavanca, agravando-se a mordida aberta anterior nos casos de ressecção de cabeça da mandíbula bilateral ou, podendo provocar maior deslocamento do fragmento nos casos de fraturas.

Quanto ao número de espátulas, inicia-se com o número máximo de espátulas que couberem entre os dentes mais posteriores presentes na boca podendo ser aumentado gradativamente segundo o ganho de abertura.

- Exercícios Isométricos:

- movimento protrusivo, mantendo-se a contração na posição mais anterior da mandíbula por 10 segundos;

- lateralidade mandibular mantendo-se por 10 segundos, repetidas vezes.

$\mathrm{V}$ - Terapia miofuncional.

- Alongamento e organização da relação cabeça e tronco;

- Mastigação unilateral alternada, direcionando-se mecanismo de contração de bucinador e de lateralização da língua unilateralmente, buscando induzir movimento mandibular rotatório;

- Aumento da amplitude do movimento mandibular na fala, por meio de pistas perceptivas e visuais;

- Treino de ressonância oral estimulando aumento de espaço intra-oral posterior por depressão de dorso de língua.

A terapêutica fonoaudiológica proposta foi realizada em 25 sessões individuais com freqüência de uma vez por semana, durante seis meses, com solicitação de freqüência do treino em casa de três a cinco vezes por dia durante as primeiras 12 semanas. Esse número de sessões foi necessário devido ao início tardio do trabalho fonoaudiológico. A cada sessão, direcionada pelo terapeuta, deve ser conseguido ao menos $5 \mathrm{~mm}$ de aumento de amplitude de abertura mandibular máxima, constatado pela marcação da amplitude dos movimentos mandibulares em início e final da terapia. O paciente, ao realizar o treino em casa durante a semana, consegue manter estabilidade desse ganho de amplitude em torno de $3 \mathrm{~mm}$. Obtendo-se amplitude máxima próxima de $35 \mathrm{~mm}$, o treino pode passar a ser realizado três vezes ao dia.

Após essa etapa de 25 sessões semanais, foi mantido acompanhamento para verificação da manutenção dos resultados com freqüência de uma vez a cada dois meses.

O levantamento dos dados desse trabalho foi iniciado após procedimentos éticos pertinentes: avaliação e aprovação pelo Comitê de Ética em Pesquisa da Instituição de origem, sob o protocolo número 81/06 e assinatura do Termo de Consentimento Livre e Esclarecido.

\section{RESULTADOS}

Os resultados quanto à evolução da abertura da boca para o caso em questão podem ser visibilizados na Figura 1.

Os resultados gerais obtidos foram:

- Redução dos mecanismos compensatórios:

- Redução da mordida aberta anterior em 4 mm;

- Ganho de $15 \mathrm{~mm}$ na abertura mandibular máxima, passando de $25 \mathrm{~mm}$ para $40 \mathrm{~mm}$;

- Estabilidade na amplitude máxima dos movimentos mandibulares conseguida e maior liberdade nos movimentos mandibulares;

- Aumento da força mastigatória;

- Melhora na articulação da fala;

- Melhores condições alimentares e de higienização oral;

- Melhora na estética e consequentemente aumento da auto-estima, constatado por depoimento espontâneo do paciente. 


\begin{tabular}{l|l}
\hline No exame: & $25 \mathrm{~mm}$ \\
\hline $2^{a}$ Semana: & $27 \mathrm{~mm}$ \\
\hline $3^{a}$ semana: & $30 \mathrm{~mm}$ \\
\hline $4^{\text {a }}$ semana: & $31 \mathrm{~mm}$ \\
\hline $5^{a}$ a $10^{a}$ semana: & $33 \mathrm{~mm}$ a $38 \mathrm{~mm}$ \\
\hline $11^{\mathrm{a}}$ a $24^{\mathrm{a}}$ semana: & $38 \mathrm{~mm}$ a $40 \mathrm{~mm}$ \\
\hline $25^{a}$ a $48^{a}$ semana: & $39 \mathrm{~mm}$ a $40 \mathrm{~mm}$ \\
\hline
\end{tabular}

Figura 1 - Tabela de Evolução da abertura da boca $(\mathrm{mm})$ com a progressão da terapia fonoaudiológica

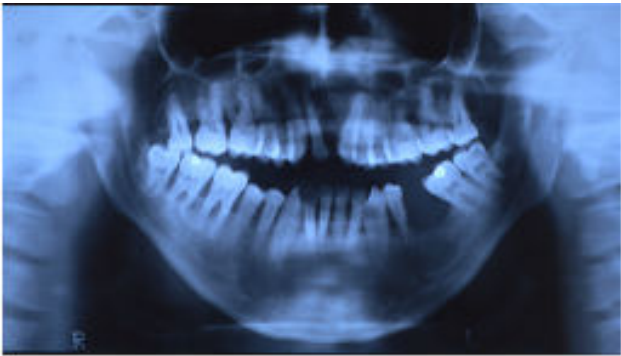

Figuras 2 - Radiografia panorâmica prévia à cirurgia, mostrando área anquilótica bilateral

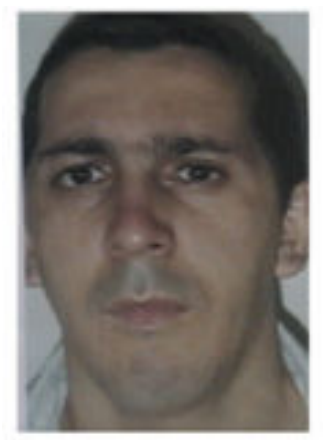

Figura 3 - Aspecto facial do paciente antes do tratamento

\section{DISCUSSÃO}

Vários métodos têm sido discutidos para prevenir as recidivas cirúrgicas de anquilose da articulação temporomandibular ${ }^{8-10,13,17,20,21}$, com relatos de alta incidência de recidiva em casos operados ${ }^{27,28}$. O sucesso do tratamento, segundo alguns autores, depende fundamentalmente da utilização da cinesioterapia precoce e quase imediata ${ }^{15,29-32}$ e de exercícios visando o aumento gradativo da abertura da boca ${ }^{20,29,30}$, apesar de que alguns trabalhos mais antigos não referem tal necessidade terapêutica ${ }^{7,23,27,34}$ provavelmente por não haver, na época, equipes com profissionais desenvolvendo essa abordagem.

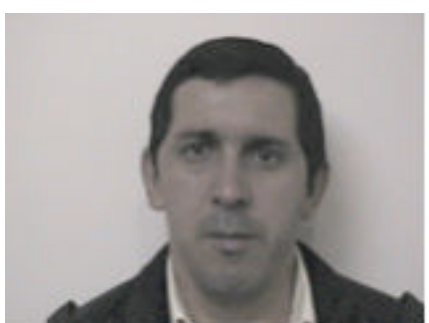

Figura 4 - Aspecto facial do paciente após o tratamento

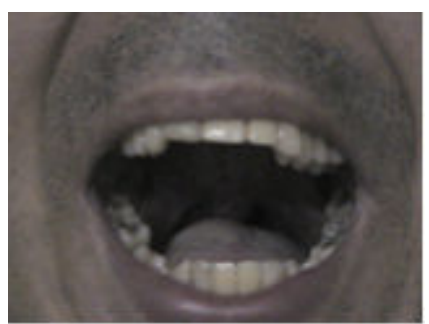

Figura 5 - Abertura da boca máxima conseguida após o tratamento fonoaudiológico e da reabilitação dentária.

A artroplastia, a qual o caso apresentado submeteuse, repousa sobre dois princípios fundamentais: realizar liberação cirúrgica adequada e promover a abertura da boca pós-operatória precoce, vigorosa e contínua ${ }^{30}$.

Dentro da proposta em restabelecer a funcionalidade do sistema estomatognático, foi desenvolvido uma programação específica envolvendo manobras e manipulações na região orofacial e cervical procurando-se respeitar as limitações do paciente em função do quadro apresentado. A região cervical foi trabalhada intensamente durante o processo terapêutico devido aos mecanismos adaptativos compensatórios utilizados pelo paciente na tentativa de realizar eficientemente as funções estomatognáticas. Movimentos envolvendo a muscula- 
tura cervical como elevação, anteriorização e rotação dos ombros; hiperextensão e hiperflexão da cabeça foram estimulados. Massagens, alongamento e manipulações nos músculos cervicais; esternocleidomastóideos e trapézio buscaram promover a oxigenação dos mesmos e redução dos resíduos metabólicos, levando a organização, mesmo que parcial, dos grupos musculares envolvidos nos mecanismos compensatórios necessários nesse tipo de caso ${ }^{31-33}$.

Considerando que a língua é uma estrutura importante de apoio e direcionamento na realização dos movimentos mandibulares devido à inserção de sua musculatura extrínseca, seus movimentos foram intensamente estimulados. Por meio de exercícios de protrusão e elevação da língua fora da boca estimula-se a abertura mandibular acompanhada por movimento mandibular protrusivo. Com o abaixamento da língua fora da boca se intensifica a abertura. A lateralização da língua, fora ou dentro da boca, estimula os movimentos de lateralidade mandibular e, sua rotação estimula os movimentos seqüenciais ${ }^{20,22,31-33}$.

A mastigação foi também trabalhada criterio-samente uma vez que atua como estímulo contínuo das estruturas trabalhadas. Propõe-se treino mastigatório com alimentos com texturas variadas e consistentes. Apenas por tratar-se de caso de anquilose temporomandibular, podem ser realizados treinos eventuais com garrote na fase final do tratamento, com o objetivo de desenvolver força mastigatória por meio de esforço dessa musculatura. Orienta-se a colocação de porções pequenas ou médias. A solicitação quanto ao padrão refere-se à utili- zação de mastigação unilateral alternada, ou seja, devese mastigar uma porção do alimento de um dos lados até sua finalização e a próxima porção deverá ser levada para o outro lado. Assim são fornecidos estímulos bilateralmente com a facilitação na organização do bolo alimentar, uma vez que os movimentos mandibulares em lateralidade são bastante precários ou inexistentes nos casos de anquilose temporomandibular bilateral ${ }^{23,31-33}$

Essa proposta interdisciplinar desenvolvida para pacientes acometidos de anquilose temporoman-dibular bilateral, visa não só aumentar e estabilizar a abertura da boca e liberar os movimentos da mandíbula após a intervenção cirúrgica, mas principalmente propiciar melhores condições de funcionalidade para esses pacientes e harmonia do complexo e dinâmico sistema estomatognático.

\section{CONCLUSÃO}

A proposta fonoaudiológica miofuncional orofacial descrita, realizada após os procedimentos cirúrgicos, mostrou-se eficiente em adulto com anquilose da articulação temporomandibular bilateral, com resultados mantidos em médio e longo prazo.

Os pacientes com anquilose temporomandibular, principalmente aqueles cujo acometimento é bilateral, requerem um processo terapêutico criterioso, rigoroso, e interdependente. Não são poucas as limitações impostas e as dificuldades que surgem típicas de cada caso e, portanto podem ser necessárias adaptações específicas aos procedimentos aqui propostos.

\begin{abstract}
Purpose: to submit an oral miofunctional therapeutic proposal, the procedures used in cases of bilateral ankylosis of the temporomandibular and the results, joint standing out the importance of interdisciplinary work and the oral miofunctional therapy contribution to rehabilitation of the stomatognatic system in these cases. Methods: this case report is about an adult, male, referred to evaluation and specific procedures three months after the joint surgery. Core complaint was great restriction of mouth opening range. The oral miofuncional exam assessment has shown: mouth opening of $25 \mathrm{~mm}$, lack of laterality and protrusion movements, previous open bite of $8 \mathrm{~mm}$, difficulty in chewing, swallowing, speech articulation and pain in temporal muscles. The therapeutical objectives were: stimulate and allow the execution of mandible movements, preventing restrictive cicatricial tissue and functional rehabilitation. Procedures related to increasing the amplitude through looseness, stretching, massages, and specific maneuvers as distraction and lever had been essential to stabilize the opening of the mouth, to liberate the mandible movements partially and to co-ordinate them as long as it improves the functionality of the stomatognatic system. Results: after 24 sessions were: gain of $16 \mathrm{~mm}$ in the opening, passing to $41 \mathrm{~mm}$ with stability according to annual control; reduction of the previous bite open for $4 \mathrm{~mm}$; improvement in the hygienic cleaning, in the speech articulation, in the alimentary conditions with acquisition of chewing strength and reduction of the compensatory mechanisms; elimination of pain in the region of the temporal muscles in addition to improvement in the facial esthetics. Conclusion: the oral miofunctional therapeutic reveals itself efficient in adult with bilateral ankylosis of the temporomandibular joint, after surgical procedure, with stability of results maintained according to annual control.
\end{abstract}

KEYWORDS: Ankylosis; Temporomandibular Joint; Stomatognathic System; Facial Injuries; Rehabilitation 


\section{REFERÊNCIAS}

1. Zemlin WR. Princípios de anatomia e fisiologia em fonoaudiologia. 4. ed. Porto Alegre: Artmed; 2000. p. 277-82.

2. Berretin G. Avaliação clínica e eletromiografica da função mastigatória em indivíduos com disfunção craniomandibular [mestrado]. Piracicaba (SP): Faculdade de Odontologia de Piracicaba da Universidade Estadual de Campinas; 1999.

3. Seroli W, Luz JGC. Anquilose da articulação têmporo-mandibular: fatores etiológicos e princípios de diagnóstico e de tratamento. Rev Bras Odontol. 1994; 51(1):41-7.

4. Vasconcelos BC, Bessa-Nogueira RV, Cypriano $\mathrm{RV}$. Treatment of temporomandibular joint ankylosis by gap arthroplasty. Med Oral Patol Oral Cir Bucal. 2006; 11(1):E66-9.

5. Luz JGC, Afonso MMV, Jorge WA. Complicações das fraturas mandibulares. Rev Gaúcha Odontol.1989; 37(4):274-8.

6. Montañez FM. Reemplazo condilar total como tratamiento de la anquilosis temporomandibular: reporte de dos casos bilaterales. Rev ADM. 2002; 59(1):34-9.

7. Kazanjian VH. Ankylosis of the temporomandibular joint. Am J Orthod. 1938; 24(12):181-206.

8. Burk JL, Newby NW, Branham GB, Provencher RF. Surgical correction of pseudoankylosis and microgenia: report of case. J Am Dent Assoc. 1977; 94(6):1173-7.

9. Hibi G, Kaneda T, Oka T. Indication and appreciation of operative procedures for mandibular ankylosis. Int J Oral Surg.1978; 7(4):333-9.

10. Hernandez RLA, Furtado JHC. Artroplastia sem material de interposição no tratamento da anquilose temporomandibular. Odontol Mod. 1986; 13(5):6-21.

11. Summers L. False ankylosis of the temporomandibular joint following craniotomy. Br J Oral Surg. 1980; 18(2):138-40.

12. Rikalainen R, Lamberg MA, Tasanen A. Extraarticular fibrous ankylosis of the mandible after zygomatic fracture. J Maxillofac Surg. 1981; 9(2):132-6.

13. Kummoona R. Functional rehabilitation of ankylosed temporomandibular joints. Oral Surg Oral Med Oral Pathol. 1978; 46(4):495-505.

14. Casanova MS, Tuji FM, Ortega AI, Yoo HJ, HaiterNeto F. Computed tomography of the TMJ in diagnosis of ankylosis: two case reports. Med Oral Patol Oral Cir Bucal. 2006; 11(5):E413-6.

15. Erol B, Tanrikulu R, Görgün B. A clinical study on ankylosis of the temporomandibular joint. $J$ Craniomaxillofac Surg. 2006; 34(2):100-6.
16. Souza LCM, Freitas RR. Anquilose da ATM. In: Barros JJ, Rode SM. Tratamento das funções craniomandibulares. São Paulo: Santos; 1995. p. 321-30.

17. Matukas VJ, Szymela VF, Schmidt JF. Surgical treatment of bony ankylosis in a child using a composite cartilage-bone iliac crest graft. J Oral Surg. 1980; 38(12):903-5.

18. Carneiro Júnior JT, Silva KBF, Grisolia DF, Félix VB. Reconstrução primária da articulação temporomandibular com enxerto costocondrial após remoção da anquilose: relato de caso. Rev Para Med. 2005; 19(1):59-63.

19. Tasanen A, Leikomaa H. Ankylosis of the temporomandibular joint of a child: report of a case. Int J Oral Surg. 1977; 6(2):95-9.

20. Bianchini EMG, Luz JGC. Nossos casos especiais. In: Bianchini EMG organizador. Articulação temporomandibular, implicações, limitações e possibilidades fonoaudiológicas. Carapicuíba: Pró-fono; 2000. p. 363-401.

21. Pacinato MNC, Genaro KF. Avaliação fonoaudiológica das funções orais de indivíduos com anquilose mandibular. J Bras Fonoaudiol. 2000; 5:77-9.

22. Bianchini EMG, Marzotto SR, Nazário D, Vicentin D. Fratura condilar em adultos: implicações e tratamento fonoaudiológico. In: IX Congresso Brasileiro de Fonoaudilogia. 2001 Set 26-29; Guarapari (ES); 2001.

23. Hellinger MJ. Bony ankylosis of the temporomandibular joint. Oral Surg Oral Med Oral Pathol. 1964; 18(3);193-302.

24. Miller GA, Page Junior HL, Griffith CR. Temporomandibular joint ankylosis: review of the literature and report two cases of bilateral involvement. J Oral Surg. 1975; 33(10):792-803.

25. Tanrikulu R, Erol B, Görgün B, Söker M. The contribution to success of various methods of treatment of temporomandibular joint ankylosis (a statistical study containing 24 cases). Turk J Pediatr. 2005; 47(3):261-5.

26. Eldirini AR. New trends in evaluation and management of recurrent ankylosis of the temporomandibular joint. Egypt Dent J. 1975; 21(3):75-88.

27. Haidar Z. Ankylosis of the tempomandibular joint. Oral Surg. 1964; 18(3):293- 302.

28. Tapazian RG. Etiology of ankylosis of the temporomandibular joint: analysis of 44 cases. J Oral Surg Anesth Hosp Dent Serv. 1964; 22:227-33.

29. Trigo JA, Zweidler EV, Pacheco DFS. Cinesioterapia associada o tratamento cirúrgico da anquilose temporomandibular. Med Reabil. 2002; (58):8-22.

30. Frankl Z, Gerendas M, Gouth J, Prohaszka I. The resolution of mandibular anklyosis. II. 
Quintessence Int Dent Dig. 1980; 11(11):9-15.

31. Bianchini EMG. Trauma de face: atuação fonoaudiológica, caracterização, proposta terapêutica e resultados. In: Comitê de Motricidade Oral da Sociedade Brasileira de Fonoaudiologia. Motricidade orofacial: como atuam os especialistas. São José dos Campos: Pulso; 2004. p. 107-14.

32. Bianchini EMG. Ajuda fonoaudiológica. In: Bianchini EMG, organizador. Articulação temporomandibular: im- plicações, limitações e possibilidades fonoaudiológicas. Carapicuíba: Pró-fono; 2000. p. 321-58.

33. Bianchini EMG, Mangilli D, Marzotto SR, Nazário D. Pacientes acometidos por trauma da face: caracterização, aplicabilidade e resultados do tratamento fonoaudiológico específico. Rev CEFAC. 2004; 6(4):388-95.

34. Dingman RD. Ankylosis of temporomandibular joint. Am J Orthod Oral Surg. 1946; 32:120-5.

RECEBIDO EM: 24/01/2007

ACEITO EM: 09/04/2007

Endereço para correspondência:

Rua Dr. Jesuíno Maciel, 1219

São Paulo - SP

CEP: 04615-003

Tel/fax: (11) 50449187

E-mail: silvanamarzoto@uol.com.br 